\title{
MicroRNAs in Colorectal Cancer Drug Resistance: Shooters become Targets
}

\author{
Daniele Fanale ${ }^{1 \dagger}$, Stefano Caruso ${ }^{1 \dagger}$, Viviana Bazan ${ }^{1}$, Giuseppe Bronte ${ }^{1}$, Florinda Di Piazza', Christian Rolfo ${ }^{2}$, Mauro Li Muli', Giuseppe \\ Cicero', and Antonio Russo ${ }^{1 *}$
}

${ }^{1}$ Department of Surgical and Oncological Sciences, Section of Medical Oncology, University of Palermo, Italy

${ }^{2}$ Oncology Department, University Hospital of Antwerp,Edegem, Antwerp, Belgium

tThese authors contributed equally to this work.

MicroRNAs (MiRNAs) are involved in the regulation of several biological processes such as development, differentiation, metabolism, apoptosis and proliferation. Recently, it has been shown that deregulated expression of miRNAs are present in different human cancers, suggesting a potential role in carcinogenesis $[1,2]$. Recent evidence suggests that miRNAs may represent potential new therapeutic approaches in patients with drug resistance and drug induced toxicity [3]. MiRNAs may have therapeutic applications through two mechanisms. The first strategy involves the inhibition of oncogenic miRNAs by using miRNA antagonists (anti-miRNAs) resulting in up-regulation of genes that would be silenced by deregulated miRNAs. The second strategy involves the restoration in expression levels of tumor suppressor microRNA with miRNA mimics [1]. To date, several studies have shown that miRNA mimics and antimiRNAs may be useful to restore normal gene networks in different cancer cell lines and animal models, suggesting a new potential role in anti-cancer therapy.

MiRNAs deregulation in Colorectal Cancer (CRC) was reported for the first time in 2003, showing the down-regulation of miR-143 and miR-145 in CRC tissues compared with normal tissues. Thereafter, numerous studies have reported different miRNA profiles in each CRC subtype [4].

Several evidences suggest that a small fraction of cells, termed Cell Stem Cells (CSCs), is responsible for the formation, growth and development of the tumor. Since this cancer cell population maintain the malignant potential and determine resistance to therapy, many tumors may progress because CSCs are resistant to cytotoxic drugs $[5,6]$. In a recent work, it has been shown that miR-451 was down-regulated in colonspheres with properties of CSCs from different colon cancer cells. Conversely, ectopic expression of miR-451 determined decreased tumorigenicity and self-renewal, and chemoresistance to irinotecan. Irinotecan causes inhibition of topoisomerase 1 by preventing DNA unwinding. Down-regulation of miR-451 increases the ATP-Binding Cassette Drug Transporter (ABCB1) resulting in irinotecan resistance. Reduced expression levels of miR-451 were detected in patients resistant to irinotecan-based first-line therapy. In 2009, it has been reported that miR-140 confers a methotrexate and 5-fluoroucil-resistant phenotype in colorectal CSC through the inibition of cell proliferation by targeting histone deacetylase 4 (HDAC4). The following year, the same authors showed that miRNA-215 enhances the chemoresistance of colorectal CSC to methotrexate and tomudex by inhibiting cell proliferation and inducing G2-arrest through the suppression of DTL expression [4].

Furthermore, Kurokawa et al. [7] detected the up-regulation of miR-19b and miR-21 in 5-fluorouracil (5FU)-resistant colon cancer cells. 5-FU resistance was related to alterations in the control of cell cycle progression caused by down-regulation of two putative gene targets: SFPQ and MYBL2 [7]. Therefore, miR-19b, miR- 21, miR140, miR-215 and miR-451 could be novel molecular markers useful to select patients resistant to therapy, but also serve as targets for the development of new possible therapeutic strategies to overcome the drug resistance.

Today, several studies focused on the use of miRNAs as possible therapeutic targets in chemotherapy resistance, by evaluating their aberrant expression in several chemoresistant tumors, in order to correct this dysregulation by means of miRNA-based drugs (antimiRNAs and miRNA mimics). This investigation is highly innovative and could have important clinical implications in understanding the molecular mechanisms responsible for chemoresistance in tumors. The identification of new possible molecular mechanisms of resistance to therapy could represent a new tool to select patients that could take benefit from this therapy. Recent advancements in the development of miRNA-based anti-cancer therapeutic approaches provided interesting results. However, despite the encouraging obtained data, the introduction of miRNAs in clinical practice is still too distant. There are still restrictions on the possible use of miRNAs as potential targets for the development of new therapeutic approaches. A possible limitation is to maintain target specificity, because miRNA targeting is sequencespecific while gene silencing requires only a partial complementarity between miRNA and mRNA. Therefore, a specific miRNA-mediated therapy could induce unwanted gene alterations. Other limiting factor is to achieve high therapeutic efficiency due to the amplitude of target gene modulation and the number of cells that can be targeted. In conclusion, further investigations are needed to specifically evaluate these approaches invarious human tumors.

\section{References}

1. Corsini LR, Bronte G, Terrasi M, Amodeo V, Fanale D et al. (2012) The role of microRNAs in cancer: diagnostic and prognostic biomarkers and targets of therapies. Expert OpinTher Targets 16 Suppl 2: S103-109.

2. McBee WC Jr, Gardiner AS, Edwards RP, Lesnock JL, et al. (2011) MicroRNA Analysis in Human Papillomavirus (HPV)-Associated Cervical Neoplasia and Cancer. J Carcinogene Mutagene 1:114.

3. Sethi S, Sarkar FH (2011) Evolving Concept of Cancer Stem Cells: Role of Micro-RNAs and their Implications in Tumor Aggressiveness. J Carcinogene Mutagene S1:005.

*Corresponding author: Antonio Russo,Section of Medical Oncology, Departmen of Surgical and Oncological Sciences, University of Palermo, Via del Vespro 129 90127 Palermo, Italy,Tel: +39-091-6552500; Fax: (011) 39-091-6554529; E-mail: mailto:antonio.russo@usa.net

Received January 22, 2013; Accepted January 25, 2013; Published February 11, 2013

Citation: Fanale D, Caruso S, Bazan V, Bronte G, Piazza FD, et al. (2013) MicroRNAs in Colorectal Cancer Drug Resistance: Shooters become Targets. J Carcinogene Mutagene 4: 136. doi:10.4172/2157-2518.1000136

Copyright: (C) 2013 Fanale D, et al. This is an open-access article distributed unde the terms of the Creative Commons Attribution License, which permits unrestricted use, distribution, and reproduction in any medium, provided the original author and source are credited. 
Citation: Fanale D, Caruso S, Bazan V, Bronte G, Piazza FD, et al. (2013) MicroRNAs in Colorectal Cancer Drug Resistance: Shooters become Targets. J Carcinogene Mutagene 4: 136. doi:10.4172/2157-2518.1000136

Page 2 of 2

4. Caruso S, Bazan V, Rolfo C, Insalaco L, Fanale D, et al. (2012) MicroRNAs in colorectal cancer stem cells: new regulators of cancer stemness? Oncogenesis 1: e32.

5. Di Fiore R, Fanale D, Drago-Ferrante R, Chiaradonna F, Giuliano M, et al (2012) Genetic and molecular characterization of the human osteosarcoma 3AB-OS cancer stem cell line: A possible model for studying osteosarcoma origin and stemness. J Cell Physiol
6. Sun L, Cabarcas SM, Farrar WL (2011) Radioresistance and Cancer Stem Cells: Survival of the Fittest. J Carcinogene Mutagene S1:004.

7. Kurokawa K, Tanahashi T, lima T, Yamamoto Y, Akaike Y, et al. (2012) Role of miR-19b and its target mRNAs in 5-fluorouracil resistance in colon cancer cells. J Gastroenterol 47: 883-895. 